\title{
PSEUDOCOMPLETE NILPOTENT GROUPS
}

\author{
THOMAS A. FOURNELLE
}

\begin{abstract}
Semicomplete nilpotent groups, that is, nilpotent groups with no outer automorphisms, have been of interest for many years. In this paper pseudocomplete nilpotent groups, that is, nilpotent groups in which the automorphism group and the inner automorphism group are isomorphic (not equal), are constructed. When suitable conditions are placed on the pseudocomplete nilpotent group, the quotient of the automorphism group by the inner automorphism group is completely characterized.
\end{abstract}

1. Introduction. Let Aut $G$ and Inn $G$ denote the group of all automorphisms of the group $G$ and the group of all inner automorphisms of $G$, respectively. A group is said to be complete if it has trivial center and if Aut $G=\operatorname{Inn} G$. A group $G$ is semicomplete if Aut $G=\operatorname{Inn} G$. While examples of complete groups abound, semicomplete groups with large centers are relatively rare. For example, no abelian group with more than two elements is ever semicomplete. Indeed, periodic nilpotent groups with more than two elements can never be semicomplete (Gaschütz [3], Zalesskii [9]). Also, nontrivial finitely generated torsion-free nilpotent groups are never semicomplete (Ree [5]). However, semicomplete nilpotent groups do exist. The first was constructed by Zalesskii [10] in 1972. Subsequently, other examples have been provided by Heinekin [4], Robinson [6], and Fournelle [1].

The purpose of this note is to describe some classes of pseudocomplete groups, that is, groups $G$ for which Aut $G \simeq \operatorname{Inn} G$. As with semicomplete groups there can be no abelian pseudocomplete group with order more than two. However, we will prove the following.

TheOREM A. Let $A$ be a finite abelian group. Then there are uncountably many nonisomorphic nilpotent groups $G$ of class two such that

(i) Aut $G \simeq \operatorname{Inn} G$,

(ii) Aut $G / \operatorname{Inn} G \simeq A \oplus A$.

All of the examples $G$ of Theorem A are torsion-free, $G / Z$ and $Z(G)$ are direct sums of torsion-free abelian groups of rank one and $Z(G) / G^{\prime}$ is periodic. Moreover, the types of the summands of $G / Z(G)$ are pairwise imcomparable. We will also prove the following.

THEOREM B. Suppose $G$ is a torsion-free nilpotent group of class two with $G / Z(G)$ and $Z(G)$ direct sums of torsion-free abelian groups of rank one and $Z(G) / G^{\prime}$ is

Received by the editors August 7, 1982.

1980 Mathematics Subject Classification. Primary 20F28; Secondary 20F18.

'Partially supported by University of Alabama RGC grant no. 1075.

(c)1983 American Mathematical Society $0002-9939 / 82 / 0000-1092 / \$ 02.75$ 
periodic. Suppose also that the types of the rank 1 summands of $G / Z(G)$ are pairwise imcomparable. If Aut $G \sim \operatorname{Inn} G$ then there is a finite abelian group $A$ such that Aut $G / \operatorname{Inn} G \simeq A \oplus A$.

The proof of Theorem B relies heavily on the theory of torsion-free abelian groups of rank one as described, for example, in Fuchs [2]. The construction of the examples in Theorem A rests upon cohomological methods as described in Stammbach [7], Warfield [8], and Robinson [6].

\section{Notation.}

$Z, Z(G)$ : the center of the group $G$,

$Q=G / Z$,

$G^{\prime}$ : the commutator subgroup of $G$,

$C_{n}, C(n)$ : the cyclic group of order $n$,

$\operatorname{Cr} G_{i}$ : the cartesian (unrestricted) product of the groups $G_{i}$,

$\operatorname{Dr} G_{i}$ : the (restricted) direct product of the groups $G_{i}$,

$\oplus G_{i}$ : additive notation for $\operatorname{Dr} G_{i}$,

$[x, y]=x^{-1} y^{-1} x y$,

$\tau(A)$ : the type of the torsion-free abelian group $A$.

2. Cohomological methods. Let $Q$ and $Z$ be abelian groups where $Z$ is regarded as a trivial $Q$-module. Let $\alpha \in$ Aut $A$ and $\beta \in$ Aut $Q$. Then $\alpha$ and $\beta$ induce automorphisms $\alpha_{*}$ and $\beta^{*}$ of $H^{2}(Q, Z)$. If $Z>G \rightarrow Q$ is a central extension with cohomology class $\Delta$, then there is an automorphism $\gamma$ of $G$ inducing $\alpha$ and $\beta$ if and only if $\Delta \alpha_{*}=\Delta \beta^{*}$ (Stammbach [7, Proposition II.4.3]). Thus we may study the automorphisms of a central extension if we have sufficient knowledge of $H^{2}(Q, Z)$. A useful tool for gaining this knowledge is the Universal Coefficients Theorem which states that there is an exact sequence

$$
\operatorname{Ext}(Q, Z)>H^{2}(Q, Z) \stackrel{\theta}{\rightarrow} \operatorname{Hom}\left(H_{2} Q, Z\right)
$$

We may identify the Schur multiplicator $\mathrm{H}_{2} Q$ with the skew-symmetric tensor product $Q \wedge Q$. Thus, $\operatorname{Hom}\left(\mathrm{H}_{2} Q, \mathrm{Z}\right)$ may be identified with the skew-symmetric bilinear forms from $Q \times Q$ to $Z$. (See Warfield [8. Theorem 5.4].)

Let $Z>G \rightarrow Q$ be a central extension with cohomology class $\Delta$. Then $\theta$ in (2.1) is the map that sends $\Delta$ to $\psi \in \operatorname{Hom}\left(H_{2} Q, Z\right)$ where

$$
\left(x^{\eta} \wedge y^{\eta}\right) \psi=[x, y] ; \quad x, y \in G .
$$

Here, of course, $[x, y]$ denotes the commutator $x^{-1} y^{-1} x y$. In our construction we will first choose a nondegenerate form $\psi$. Then we will choose $\bar{\Delta}$ to be a preimage of $\psi$ under $\theta$. Let $\sigma$ be an element of $\operatorname{Ext}(Q, Z)$. We may alter $\bar{\Delta}$ by defining $\Delta=\bar{\Delta}+\sigma$. Note that $\Delta \theta=\bar{\Delta} \theta=\psi$ and any extension with class $\Delta$ will be strictly central (that is, $Z$ will be exactly the center of $G$ ) since $\psi$ is nondegenerate.

Now if $f: Q \times Q \rightarrow Z$ is a 2-cocycle, define $(x, y) f^{\prime}=(y, x) f$. Then the map $f \mapsto f^{\prime}$ is an automorphism of $Z^{2}(Q, Z)$, the group of all 2-cocycles, which fixes coboundaries. Thus, there is an induced automorphism $\Delta \mapsto \Delta^{\prime}$ of $H^{2}(Q, Z)$. For 
any $\Delta \in H^{2}(Q, Z)$ we define $\Delta_{s}=\Delta+\Delta^{\prime}$. Then $\Delta_{s} \in \operatorname{Ext}(Q, Z)$, and if $\alpha \in$ Aut $Z$ and $\beta \in$ Aut $Q$, then $\Delta \alpha_{*}=\Delta \beta^{*}$ implies that $\Delta_{s} \alpha_{*}=\Delta_{s} \beta^{*}$ and $(\Delta \theta) \alpha_{*}=(\Delta \theta) \beta^{*}$. Also, if $\bar{\Delta} \in H^{2}(Q, Z), \sigma \in \operatorname{Ext}(Q, Z)$, and $\Delta=\bar{\Delta}+\sigma$, then

$$
\Delta_{s}=\bar{\Delta}_{s}+2 \sigma \text {. }
$$

We will need all of the above to determine the structure of our examples.

We also will need to be able to compute $\operatorname{Ext}(A, B)$ where $A$ and $B$ are certain rank one torsion-free abelian groups. In particular, let $\pi$ be an infinite set of primes and let $\rho$ be an infinite subset. Now consider the following two subgroups of the rational numbers:

$$
A=\langle 1 / p: p \in \rho\rangle, \quad B=\langle 1 / p: p \in \pi\rangle .
$$

From the short exact sequence $\mathbf{Z} \longrightarrow A \rightarrow A / \mathbf{Z}$ we obtain a long exact sequence (Fuchs [2, Vol. 1, IX])

$$
0 \rightarrow \operatorname{Hom}(A, B) \rightarrow \operatorname{Hom}(\mathbf{Z}, B) \rightarrow \operatorname{Ext}(A / \mathbf{Z}, B) \rightarrow \operatorname{Ext}(A, B) \rightarrow 0 .
$$

Hence there is a short exact sequence

$$
C>\operatorname{Ext}(A / \mathbf{Z}, B) \rightarrow \operatorname{Ext}(A, B)
$$

where $C$ is countable and of torsion-free rank at most one. Now, $A / \mathbf{Z} \sim \oplus_{p \in}{ }_{p} C_{p}$ and thus

$$
\operatorname{Ext}(A / \mathbf{Z}, B) \simeq \underset{p \in \rho}{\operatorname{Cr}} C_{p} .
$$

The sequence $C \longrightarrow \mathrm{Cr}_{p \in \rho} C_{p} \rightarrow \operatorname{Ext}(A, B)$ now yields the fact that $\operatorname{Ext}(A, B)$ is of uncountable torsion-free rank.

3. Proof of Theorem A. Let $\pi$ and $\rho$ be two disjoint infinite sets of primes. Let $q$ be a fixed prime with $q \notin \pi \cup \rho$. Consider the following rational groups:

$$
Q_{1}=\langle 1 / p: p \in \pi\rangle, \quad Q_{2}=\langle 1 / p: p \in \rho\rangle, \quad Z=\langle 1 / p: p \in \pi \cup \rho\rangle .
$$

Let $Q=Q_{1} \oplus Q_{2}$. We choose $\psi: Q \times Q \rightarrow Z$ to be the skew-symmetric bilinear form given by $((a, b),(c, d)) \psi=q^{n}(a d-b c)$. Note that $\psi$ is nondegenerate. Let $\bar{\Delta}$ be a preimage of $\psi$ under $\theta$ in (2.1). Now $\operatorname{Ext}(Q, Z) \simeq \operatorname{Ext}\left(Q_{1}, Z\right) \oplus \operatorname{Ext}\left(Q_{2}, Z\right)$ and each $\operatorname{Ext}\left(Q_{i}, Z\right)$ is of infinite torsion-free rank. Hence, by (2.2) we may choose a preimage $\Delta$ of $\psi$ such that $\Delta_{s}=\left(f_{1}, f_{2}\right)$, where $f_{i} \in \operatorname{Ext}\left(Q_{i}, Z\right)$ and where $f_{1}$ and $f_{2}$ are of infinite order and linearly independent in $\operatorname{Ext}(Q, Z)$. Let $Z>G \rightarrow Q$ be a central extension with cohomology class $\Delta$. Then $G$ is torsion-free and nilpotent of class two and $Z$ is the center of $G$ since $\psi$ is nondegenerate.

Lemma 3.1. Let $\gamma \in$ Aut $G$. Then $\gamma$ induces the identity on $Q$ and $Z$.

Proof. Suppose that $\gamma$ induces $\alpha$ on $Z$ and $\beta$ on $Q$. Then $\Delta \alpha_{*}=\Delta \beta^{*}$. Since $Z$ is torsion-free of rank one it follows that $\alpha$ is multiplication by some rational number $t$. However, the denominators in $Z$ are square free and thus $t= \pm 1$. Since $Q_{1}$ and $Q_{2}$ are characteristic in $Q$ we may represent $\beta$ by the matrix $\left(\begin{array}{ll}u & 0 \\ 0 & v\end{array}\right)$ where $u$ and $v$ are rationals. Again $Q_{1}$ and $Q_{2}$ have square free denominators so $u= \pm 1$ and $v= \pm 1$. Now, $\Delta_{s} \alpha_{*}=\Delta_{s} \beta^{*}$ implies $\left(f_{1}, f_{2}\right) \alpha_{*}=\left(t f_{1}, t f_{2}\right)=\left(f_{1}, f_{2}\right) \beta^{*}=\left(u f_{1}, v f_{2}\right)$ [2, Vol. 1, Lemma 52.1]. Hence $t=u=v= \pm 1$. Also, $\psi \alpha_{*}=\psi \beta^{*}$. Hence, if $a, b \in G$ we let 
$\bar{a}, \bar{b}$ be their images in $Q$. Suppose $\bar{a} \in Q_{1}$ and $\bar{b} \in Q_{2}$. Then $[a, b] \alpha=(\bar{a} \beta, \bar{b} \beta) \psi$. Hence, $t[a, b]=[u a, v b]=u v[a, b]$ and therefore $t=u v$. Consequently, $t^{2}=t$ and $t=u=v=1$. This completes the proof.

Now let $\gamma \in$ Aut $G$. Since $\gamma$ induces the identity on $Q$ it follows that for every $g \in G$ there is some $z_{g} \in Z$ such that $g \gamma=g z_{g}$. Since $\gamma$ induces the identity on $Z$, the mapping $g \mapsto z_{g}$ is a homomorphism from $G$ to $Z$ which contains $Z$ in its kernel. Hence, we may associate with $\gamma$ a homomorphism from $Q$ to $Z$. This association establishes the isomorphism

$$
\text { Aut } G \simeq \operatorname{Hom}(Q, Z) \text {. }
$$

Furthermore,

$$
\operatorname{Hom}(Q, Z) \simeq \operatorname{Hom}\left(Q_{1}, Z\right) \oplus \operatorname{Hom}\left(Q_{2}, Z\right) \simeq Q_{2} \oplus Q_{1} \simeq Q .
$$

Thus, Aut $G \simeq \operatorname{Inn} G$, and hence $G$ is pseudocomplete. It remains to determine Aut $G / \operatorname{Inn} G$.

Let $\gamma$ be an automorphism of $G$ corresponding to $\theta \in \operatorname{Hom}(Q, Z)$. Let $A=(1,0) \theta$ and $B=(0,1) \theta$. Then $(a, b) \theta=a A+b B$. Since homomorphisms from $Q_{1}$ and $Q_{2}$ to $Z$ are multiplication by some rational number, and since denominators in $Z$ are square free, it follows that $A \in Q_{2}$ and $B \in Q_{1}$. Hence,

$$
q^{n}(a, b) \theta=((a, b),(-B, A)) \psi .
$$

It follows that if $m=q^{n}$, then $\gamma^{m}$ is induced from conjugation in $G$ by a preimage of $(-B, A)$. It now also follows that Aut $G / \operatorname{Inn} G \simeq Q / m Q \simeq C_{m} \oplus C_{m}$.

Now suppose that $A=\operatorname{Dr}_{i=1}^{n} C_{m(i)}$ is a finite abelian group where $m(i)=p_{i}^{n(i)}$ for various primes $p_{i}$. For each $i$ we construct a pseudocomplete nilpotent group $G_{i}$ with Aut $G_{i} / \operatorname{Inn} G_{i} \simeq C_{m(i)} \oplus C_{m(i)}$. In this construction we may choose the primes involved so that the types of the factors in $G_{i} / Z_{i}$ are incomparable with the types of the factors in $G_{j} / Z_{j}$ for $i \neq j$. Thus, $\operatorname{Hom}\left(G_{i}, G_{j}\right)=0$ for $i \neq j$. We now set $G=\operatorname{Dr}_{i=1}^{n} G_{i}$. Hence, each $G_{i}$ is characteristic in $G$ and by restricting automorphisms of $G$ to the $G_{i}$ factors we are led to the isomorphisms

$$
\text { Aut } G \simeq \operatorname{Dr}_{i=1}^{n} \text { Aut } G_{i}, \quad \operatorname{Inn} G \simeq \operatorname{Dr}_{i=1}^{n} \operatorname{Inn} G_{i} \simeq \operatorname{Dr}_{i=1}^{n} \text { Aut } G_{i}
$$

It is now clear that

$$
\text { Aut } G / \operatorname{Inn} G \simeq \operatorname{Dr}_{i=1}^{n}\left(\text { Aut } G_{i} / \operatorname{Inn} G_{i}\right) \simeq A \oplus A
$$

and $G$ is a pseudocomplete torsion-free nilpotent group of class two.

REMARK 1. Suppose that there are only countably many isomorphism classes of groups $G$ constructed above. If $\bar{G}$ has class $\bar{\Delta} \in H^{2}(Q, Z)$ and $G \simeq \bar{G}$ then $\Delta \alpha_{*}=$ $\bar{\Delta} \beta^{*}$ for some $\alpha \in$ Aut $Z$ and some $\beta \in$ Aut $Q$ (Stammbach [7, Proposition II. 4.3]). However, Aut $Q$ and Aut $Z$ are finite and hence these extensions fall into countably many classes in $H^{2}(Q, Z)$. But since $\operatorname{Ext}(Q, Z)$ has uncountable torsion-free rank we have uncountably many choices for $\sigma$ in (2.2) and hence for $\Delta$. Thus, we have uncountably many choices for $G$. 
REMARK 2 . There are uncountably many rational groups of incomparable types in which the denominators of all elements are square free. Hence, for each $i$ in an uncountable index set $I$ we may construct a pseudocomplete nilpotent group $G_{i}$ with Aut $G_{i} / \operatorname{Inn} G_{i}=C_{m(i)} \oplus C_{m(i)}$. If we let $G=\mathrm{Cr}_{i \in I} G_{i}$ we have

$$
\begin{gathered}
\text { Aut } G \simeq \underset{i \in I}{\operatorname{Cr}} \text { Aut } G_{i} \simeq \underset{i \in I}{\operatorname{Cr}} \operatorname{Inn} G_{i} \simeq \operatorname{Inn} G . \\
\text { Aut } G / \operatorname{Inn} G \simeq \underset{i \in I}{\operatorname{Cr}}\left(C_{m(i)} \oplus C_{m(i)}\right) .
\end{gathered}
$$

Hence, there are pseudocomplete nilpotent groups of large rank in which Aut $G / \operatorname{Inn} G$ is large.

REMARK 3. If we choose the finite abelian group $A$ to be trivial we obtain semicomplete nilpotent groups $G$, that is, Aut $G=\operatorname{Inn} G$.

REMARK 4. The techniques used to construct the examples of this section are taken from Robinson's (unpublished) paper [6]. The author wishes to thank Professor Robinson for his kind permission to make use of these techniques here.

4. Proof of Theorem B. Now assume that $G$ is a torsion-free nilpotent group of class two with center $Z$. Assume further that $Q=G / Z$ and $Z$ are direct sums of torsion-free abelian groups of rank one and that $G / Z^{\prime}$ is periodic. Finally, assume that the types of the summands in $Q$ are pairwise incomparable. It is fairly easy to see that Aut $G / \operatorname{Inn} G$ is a finite abelian group (see [2, Vol. 2, p. 153, Example 5]). We must show that Aut $G / \operatorname{Inn} G \simeq A \oplus A$ where $A$ is a finite abelian group. We will be assuming many results from [2, Vol. 2, §85].

Lemma 4.1. Let $\gamma \in$ Aut $G$ where $G$ is as above. Then $\gamma$ induces the identity on $Z$ and on $Q$.

Proof. Let $\gamma \in$ Aut $G$ and let $\tau_{g}$ be the inner automorphism of $G$ induced by $g$. Let $x \in G$. Since Aut $G \simeq Q$ is abelian it follows that $(x) \gamma \tau_{g}=(x) \tau_{g} \gamma$. Consequently, $(g) \gamma g^{-1}$ centralizes $(x) \gamma$ for all $x \in G$. Thus, $(g) \gamma \equiv g(\bmod Z)$, that is, $\gamma$ induces the identity on $Q$. Clearly, $[a, b]=[(a) \gamma,(b) \gamma]=[a, b] \gamma$ and thus $\gamma$ induces the identity of $G^{\prime}$ also.

Now let $x \in Z$. Since $Z / G^{\prime}$ is periodic there is some nonzero $m$ such that $x^{m} \in G^{\prime}$. Thus, $\left(x^{m}\right) \gamma=x^{m}=(x \gamma)^{m}$. However, $Z$ is a torsion-free abelian group and consequently $x \gamma=x$. This completes the proof.

We now write $Q=\bigoplus_{i=1}^{n} Q_{i}$ where the $Q_{i}$ are the rank one summands of $Q$. Note that the types of these summands are invariants of $Q$ [2, Vol. 2, Proposition 86.1]. Hence any two decompositions of $Q$ into a direct sum of rank one groups are equivalent. Now from Lemma 4.1 it follows that $\operatorname{Aut} G \simeq \operatorname{Hom}(Q, Z)$. Since $G$ is pseudocomplete we have

$$
Q \simeq \operatorname{Hom}(Q, Z) \simeq \bigoplus_{i=1}^{n} \operatorname{Hom}\left(Q_{i}, Z\right)
$$

Now if $a, b \in Q$ we will let $[a, b]$ denote the commutator $\left[a_{1}, b_{1}\right]$ where $a_{1}$ and $b_{1}$ are preimages in $G$ of $a$ and $b$, respectively. Also, $\left[Q_{i}, Q_{j}\right]$ will have a similar meaning. Since congruence modulo $Z$ leaves commutators unaffected there should 
be no confusion. Note that in a group of class two, commutators are homomorphic in each variable. We also write $Z=\bigoplus_{k} Z_{k}$ where the $Z_{k}$ are the rank one summands of $Z$.

LEMMA 4.2. For each $i=1,2, \ldots, n$ there are unique $j$ and $m$ such that $\left[Q_{i}, Q_{j}\right]$ and $\operatorname{Hom}\left(Q_{i}, Z_{m}\right)$ are nontrivial. Moreover for such $i, j$, and $m$ we have

$$
\tau\left(Q_{j}\right)=\tau\left(\operatorname{Hom}\left(Q_{i}, Z_{m}\right)\right)=\tau\left(\operatorname{Hom}\left(Q_{i}, Z\right)\right)
$$

Proof. Certainly there is some $j$ such that $\left[Q_{i}, Q_{j}\right] \neq 0$ for otherwise $Q_{i}$ is central. Hence, there is some $a \in Q_{j}$ such that the commutator $[-, a]$ induces a nontrivial homomorphism from $Q_{i}$ to $Z$. From (4.1) it follows that $\operatorname{Hom}\left(Q_{i}, Z\right)$ is of rank one. However we have

$$
\operatorname{Hom}\left(Q_{i}, Z\right)=\operatorname{Hom}\left(Q_{i}, \bigoplus_{k} Z_{k}\right) \simeq \bigoplus_{k} \operatorname{Hom}\left(Q_{i}, Z_{k}\right)
$$

Since the summands on the right of (4.2) are of rank 0 or 1 , it follows that there is a unique $m$ such that $\operatorname{Hom}\left(Q_{i}, Z_{m}\right) \neq 0$.

Now for $a \in Q_{j}$ and $x \in Q_{i}$ consider $(x) \theta_{a}=[x, a]$. The mapping $a \mapsto \theta_{a}$ is a monomorphism from $Q_{j}$ to $\operatorname{Hom}\left(Q_{i}, Z_{m}\right)$. Thus,

$$
\tau\left(Q_{j}\right) \leqslant \tau\left(\operatorname{Hom}\left(Q_{i}, Z_{m}\right)\right)=\tau\left(\operatorname{Hom}\left(Q_{i}, Z\right)\right) .
$$

However, $Q_{j}$ and $\operatorname{Hom}\left(Q_{i}, Z\right)$ are rank one summands of $Q$ and the types of these summands are pairwise incomparable. Hence, $\tau\left(Q_{j}\right)=\tau\left(\operatorname{Hom}\left(Q_{i}, Z\right)\right)$ and the result now follows.

We have an immediate corollary.

COROllary 4.3. $Q$ is of even rank.

We now reindex the $Q_{i}$, if necessary, so that $\left[Q_{2 i-1}, Q_{2 i}\right] \neq 0$ for $i=1,2, \ldots, n / 2$. The mapping $a \mapsto[-, a]$ induces injections

$$
Q_{2 i-1}>\operatorname{Hom}\left(Q_{2 i}, Z\right) \text { and } Q_{2 i}>\operatorname{Hom}\left(Q_{2 i-1}, Z\right) \text {. }
$$

We then have the following isomorphisms:

$$
\text { Aut } \begin{aligned}
G / \operatorname{Inn} G & \simeq \operatorname{Hom}(Q, Z) / Q \\
& \simeq\left[\bigoplus_{i}\left(\operatorname{Hom}\left(Q_{2 i-1}, Z\right) / Q_{2 i}\right)\right] \oplus\left[\bigoplus_{i}\left(\operatorname{Hom}\left(Q_{2 i}, Z\right) / Q_{2 i-1}\right)\right] .
\end{aligned}
$$

The next result will complete the proof of Theorem $\mathrm{B}$.

LEMMA 4.4. $\operatorname{Hom}\left(Q_{2 i}, Z\right) / Q_{2 i-1} \simeq \operatorname{Hom}\left(Q_{2 i-1}, Z\right) / Q_{2 i}$.

Proof. To simplify notation we will consider only $Q_{1}$ and $Q_{2}$, and will assume $\operatorname{Hom}\left(Q_{1}, Z_{1}\right) \neq 0 \neq \operatorname{Hom}\left(Q_{2}, Z_{1}\right)$. Since $\tau\left(Q_{1}\right)=\tau\left(\operatorname{Hom}\left(Q_{2}, Z_{1}\right)\right)$ and $\tau\left(Q_{2}\right)=$ $\tau\left(\operatorname{Hom}\left(Q_{1}, Z_{1}\right)\right)$, it follows that the quotient groups we are considering are cyclic. Thus, suppose $\phi$ generates $\operatorname{Hom}\left(Q_{2}, Z_{1}\right)=\operatorname{Hom}\left(Q_{2}, Z\right)$ modulo $Q_{1}$. Suppose further that $\phi$ is of order $n$ modulo $Q_{1}$. Suppose that $a$ and $b$ are nontrivial elements of $Q_{1}$ and $Q_{2}$, respectively. Then for some $r / s$ with $(r, s)=1$ we have

$$
n(b) \phi=[b,(r / s) a]=(r / s)[b, a] \text {. }
$$


Clearly $n$ and $r$ are relatively prime for otherwise $\phi$ has order, modulo $Q_{1}$, smaller than $n$. Hence, $(n s, r)=1$ and there are integers $u, v$ such that $u n s+v r=1$. Thus,

$$
[b, a]=n s(u[b, a]+(v r / n s)[b, a])
$$

and consequently $[b, a]$ is divisible by $n$ in $Z_{1}$.

Now suppose $(u / v) a \in Q_{1}$, for some $u / v$ with $(u, v)=1$. It can be shown as above that $[b,(u / v) a]$ is divisible by $n$ in $Z_{1}$. We may thus define a homomorphism

$$
\theta: Q_{1} \rightarrow Z_{1}, \quad(u / v) a \mapsto n^{-1}[b,(u / v) a] .
$$

Modulo $Q_{2}$ the order of $\theta$ is $n$. Hence, the cyclic group $\operatorname{Hom}\left(Q_{2}, Z\right) / Q_{1}$ has order no larger than the cyclic group $\operatorname{Hom}\left(Q_{1}, Z\right) / Q_{2}$. By symmetry these two groups have the same order and thus are isomorphic. This completes the proof.

\section{REFERENCES}

1. T. A. Fournelle, Torsion in semicomplete nilpotent groups (to appear)

2. L. Fuchs, Infinite Abelian groups, Academic Press, New York and London, 1970, 1973.

3. W. Gaschütz, Nichtabelsche p-Gruppen hesitzen äussere p-Automorphismen, J. Algebra 4 (1966), 1-2.

4. H. Heineken, Automorphism groups of torsion-free nilpotent groups of class 2, Symposia Math. 17 (1976), 235-250.

5. R. Ree. The existence of outer automorphisms of some groups, Proc. Amer. Math. Soc. 7 (1956), 962-964.

6. D. J. S. Robinson, Outer automorphisms of torsion-free nilpotent groups, unpublished.

7. U. Stammbach, Homology in group theory, Lecture Notes in Math., vol. 359, Springer-Verlag, Berlin, 1972.

8. R. B. Warfield, Nilpotent groups, Lecture Notes in Math., vol. 513, Springer-Verlag, Berlin, 1976.

9. A. E. Zalesskiī, A nilpotent p-group possesses an outer automorphism, Dokl. Akad. Nauk SSSR 196 (1971), 751-754 = Soviet Math. Dokl. 12 (1972), 227-230.

10. An example of a torsion-free nilpotent group having no outer automorphisms, Mat. Zametki 11 (1972), 21-26 = Math Notes 11 (1972), 16-19.

Department of Mathematics, University of Alabama, University, Alabama 35486 